\title{
Decreased regional left ventricular myocardial strain in type 1 diabetic children: A first sign of diabetic cardiomyopathy?
}

\author{
Amir Hodzic ${ }^{1}$, Virginie Ribault ${ }^{2}$, Pascale Maragnes ${ }^{1}$, Paul Milliez ${ }^{1}$, Eric Saloux ${ }^{1}$, \\ Fabien Labombarda ${ }^{1}$ \\ 'Department of Cardiology, Caen CHU, Caen, F-14000, France; \\ 2Department of Pediatrics, Caen CHU, Caen, F-14000, France
}

\section{ABSTRACT}

Background and Objectives: Type 1 diabetes is a major cardiovascular risk factor associated with an excess of mortality in young adults due to premature cardiovascular events, which includes heart failure. The relation between type 1 diabetes and cardiac structure and function in children was poorly documented. Our study investigates (1) whether type 1 diabetic children have echocardiographic signs of subclinical cardiac dysfunction assessed by tissue Doppler strain and (2) whether state of metabolic control and diabetes duration have any influence on the cardiac event. Methods: Standard echocardiography and tissue Doppler imaging were prospectively performed in type 1 diabetic children. Left ventricular dimensions, standard indices of systolic and diastolic function, and septal longitudinal strain were investigated. Results: Thirty consecutive asymptomatic diabetic children (age: 12.4 [5-17] years; males: $53 \%$ ) were compared to 30 age and sex-matched healthy control subjects. Left ventricular mass index and diastolic septal thickness were significantly increased in diabetic children. There was no difference between two groups as regards the left ventricular ejection fraction and conventional mitral Doppler parameters (E, A, Ea). The global longitudinal systolic strain and strain rate were found to be decreased in children with diabetes. The global longitudinal early diastolic strain rate (Esr) was negatively correlated with metabolic control. Longitudinal strain was not correlated with diabetes duration. Conclusion: Children with Type 1 diabetes had subclinical alterations in left ventricular size and longitudinal myocardial deformation.

Key words: left ventricular size, type 1 diabetes, longitudinal myocardial deformation, children

Address for Correspondence: Dr. Fabien Labombarda, Department of Cardiology, $\mathrm{CHU}$ de Caen, Avenue Cote de Nacre, 14000 Caen,

France.

Email: fabien.labombarda@gmail.com

\begin{tabular}{|l|}
\hline Access this article online \\
\hline Website: \\
www.intern-med.com \\
\hline DOI: \\
10.1515/jtim-2016-0025 \\
\hline Quick Response Code: \\
\hline \\
\end{tabular}

\section{INTRODUCTION}

Type 1 diabetes is a major cardiovascular risk factor. Its prevalence has risen steadily for about 20 years with an increasingly early detection ${ }^{[1]}$. Currently in France, the incidence of type 1 diabetes is 13.5 per 100,000 children under 15 years old. Type 1 diabetes is associated with excess mortality in young adults due to premature cardiovascular events $^{[2]}$, including heart failure ${ }^{[3,4]}$. Heart failure is the consequence of myocardial abnormalities specific to diabetes, which occur even in the absence of coronary artery disease or high blood pressure, and is directly related to chronic hyperglycemia. Chronic hyperglycemia induces complex metabolic disturbances in cardiomyocytes, leading to morphological and functional abnormalities of the myocardium, grouped under the term "diabetic cardiomyopathy"[[]. Diabetic cardiomyopathy is characterized by the presence of extensive myocardial fibrosis, myocyte hypertrophy, increased oxidative stress, and microangiopathy ${ }^{[6]}$. While frequent, to date, a specific strategy for early detection or treatment of diabetic cardiomyopathy to improve its prognosis has not yet been established ${ }^{[6]}$. In adults, echocardiographic or magnetic resonance studies have shown that diabetes led to eccentric left ventricular (LV) hypertrophy with alterations of the diastolic and systolic myocardial function ${ }^{[7,8]}$. The relationship between type 1 diabetes and cardiac structure and function in children and adolescents was 
poorly documented. Most of the previous echocardiographic studies have used standard techniques relatively insensitive to the detection of early myocardial dysfunction ${ }^{[0,10]}$. Strain and strain rate, derived from tissue Doppler imaging (TDI), have been proposed as parameters for the early detection of myocardial dysfunction ${ }^{[11]}$. This technique can detect subclinical changes in myocardial function and represent an independent prognostic factor ${ }^{[12]}$. Our study investigates (1) whether children with type 1 diabetes have echocardiographic signs of subclinical cardiac dysfunction assessed by color tissue Doppler imaging and (2) whether the state of metabolic control and diabetes duration have an influence on the cardiac event.

\section{METHODS}

\section{Population study}

The study prospectively recruited patients with type 1 diabetes aged 5 to 18 years followed up at the pediatric department of the Caen Teaching Hospital. Type 1 diabetes was diagnosed according to the World Health Organization criteria ${ }^{[13]}$ together with the permanent need for insulin therapy. Exclusion criteria were the presence of cardiopathy, significant concomitant disease, medication known to modify cardiac function, high blood pressure, smoking, dyslipidemia, and obesity (defined as a body mass index (BMI), adjusted for gender and age, exceeding the 97th percentile according to French reference values ${ }^{[14]}$. Recently diagnosed ( $<1$ year) children with diabetes were not included. Patients with diabetes were compared with healthy control children from our outpatients department of pediatric cardiology selected from children being investigated for physiological cardiac murmur whose echocardiography was normal. To be included in the control group, children had to have no personal antecedents or family history of either high blood pressure or hypercholesterolemia. The study protocol was approved by the hospital ethics review board. Patients provided their informed consent through legal representatives.

\section{Clinical evaluation}

Demographic details of age, gender, weight, height, and heart rate were recorded. BMI was calculated according to the formula of weight $(\mathrm{kg})$ divided by height squared $\left(\mathrm{m}^{2}\right)$ and expressed as a $Z$ score. Systolic blood pressure (SBP) and diastolic blood pressure (DBP) were measured after 10 min at rest with a calibrated automatic blood pressure monitor (Datascope ${ }^{\circledR}$ DUO). Mean blood pressure (MBP) was calculated according the formula: $\mathrm{MBP}=(\mathrm{SBP}+$ 2xDBP)/3. SDP, DPB and MBP were expressed in $\mathrm{mmHg}$. For patients with diabetes, diabetes duration (expressed in years) was considered for each individual based on the fullattained age on the first day of insulin therapy.

\section{Biochemistry}

Fasting blood samples were taken from the diabetic children to analyze $\mathrm{HbA}_{1 \mathrm{c}}$, expressed in percentages. $\mathrm{HbA}_{1 \mathrm{c}}$ was measured by high-performance liquid chromatography (Tosoh Corporation, Tokyo, Japan). This study used the mean quarterly $\mathrm{HbA}_{1 \mathrm{c}}(\mathrm{mmol} / \mathrm{mol}, \%)$ for the year prior to the study.

\section{Echocardiography}

Echocardiographic studies were performed using an iE33 (Philips Medical Systems, S5 probe, Best, The Netherlands). Each subject was examined in a semisupine left lateral position. The electrocardiogram was recorded continuously. Images were obtained at endexpiratory apnea and stored in cineloop format from three consecutive beats.

\section{Standard Echocardiography and Tissue Doppler imaging}

Left ventricular ejection fraction (LVEF) was assessed using the biplane Simpson's method in the apical view. Left ventricular end-diastolic dimension (LV-EDD), interventricular septal end-diastolic dimension (IVSEDD), and left ventricular posterior wall end-diastolic dimension (PW-EDD) were measured in time motion mode in parasternal long-axis view. Measurements were adjusted for age, sex, height, and weight and expressed as a $Z$-score. Left ventricular mass (LVM) was calculated by the Devereux formula and indexed by height raised to the power of 2.7. The mitral Doppler signal was recorded in the apical four-chamber view, with the Doppler sample volume placed at the tip of the mitral valve. Peak velocities of early (E) and late (A) filling waves, early/late filling ratio of peak velocities (E/A) were measured on the basis of transmitral flow velocities. Myocardial systolic and diastolic velocities were recorded using the pulsed-wave TDI technique from an apical four-chamber view. The sample volume (4 mm thick) was placed at the basal level of the right ventricular and left ventricular free walls to measure tricuspid peak systolic velocity (St) and early mitral peak diastolic velocity $(\mathrm{Ea})$.

\section{Strain analysis}

For longitudinal strain and strain rate measurements, narrow sector angle acquisitions of the septal wall were obtained in the apical four-chamber view with color TDI (minimum frame rate, $90 \mathrm{~Hz}$ ) during brief apnea after expiration. Probe movements were maximally limited. Three consecutive beats were stored digitally and analyzed offline using dedicated research software (QLAB; Philips Medical Systems) capable of extracting strain and strain rate from tissue velocity data sets. During processing, manual tagging was performed, and curved M-mode lines (10-mm-thick area of interest) were drawn on the septal wall to obtain the mean strain and 
strain rate values of basal and medial septal segments. A tracking of the myocardial region of interest was activated to avoid blood flow artifacts. The peak systolic strain was determined as the nadir of the strain curves (Figure 1). The peak systolic strain rate (Ssr), early (Esr), and late (Asr) peak diastolic strain rates were measured (Figure 1). With our measurement method, strain and strain rates for the septal wall are reliable parameters for both reproducibility and repeatability, as previously reported by our team ${ }^{[15]}$. Apnea is required for color TDI acquisition and our study limit was age 5 years. In our experience, most children aged under 5 are unable to cooperate for apnea.

\section{Statistical methods}

Quantitative variables were described using mean and standard deviations (SD), and qualitative variables were described using frequencies and percentages. The two groups were compared using the two-tailed Wilcoxon test for nonparametric paired data and a paired Student's $t$-test for normally distributed paired data. A chi-square test was used to estimate the relationship between qualitative variables. Correlations between LVMD indicators and diabetes duration and $\mathrm{HBA}_{1 \mathrm{C}}$ were determined using Pearson coefficient correlation. All tests were two- tailed and their level of significance was defined as $P<$ 0.05 . $\mathrm{R}$ version 2.12 .1 was the statistical software used. The statistical review of the study was performed by a biomedical statistician.

\section{RESULTS}

\section{Population}

Overall, 30 consecutive children and adolescents with type 1 diabetes (age range [5-17] years, male: 53\%) were recruited at our pediatric endocrinology department and enrolled in this prospective cohort. Among children with diabetes, $\mathrm{HBA}_{1 \mathrm{c}}$ ranged from $6.8 \%$ to $12.7 \%, 63 \%$ of the patients with diabetes patients had $\mathrm{HBA}_{1 \mathrm{C}}>8 \%$ and mean diabetes duration was $5.1 \pm 3.1$ years. The results in diabetic patients were compared with those in 30 healthy children, matched for age and sex, recruited as a control group over the same time period. SBP was significantly higher in the diabetic group. Both weight and BMI were higher in patients with diabetes but $Z$ score BMI did not differ. The diabetic and control children were comparable with respect to age, gender, heart rate, SBP, DBP, and mean BP. The characteristics of our study population are shown in Table 1.

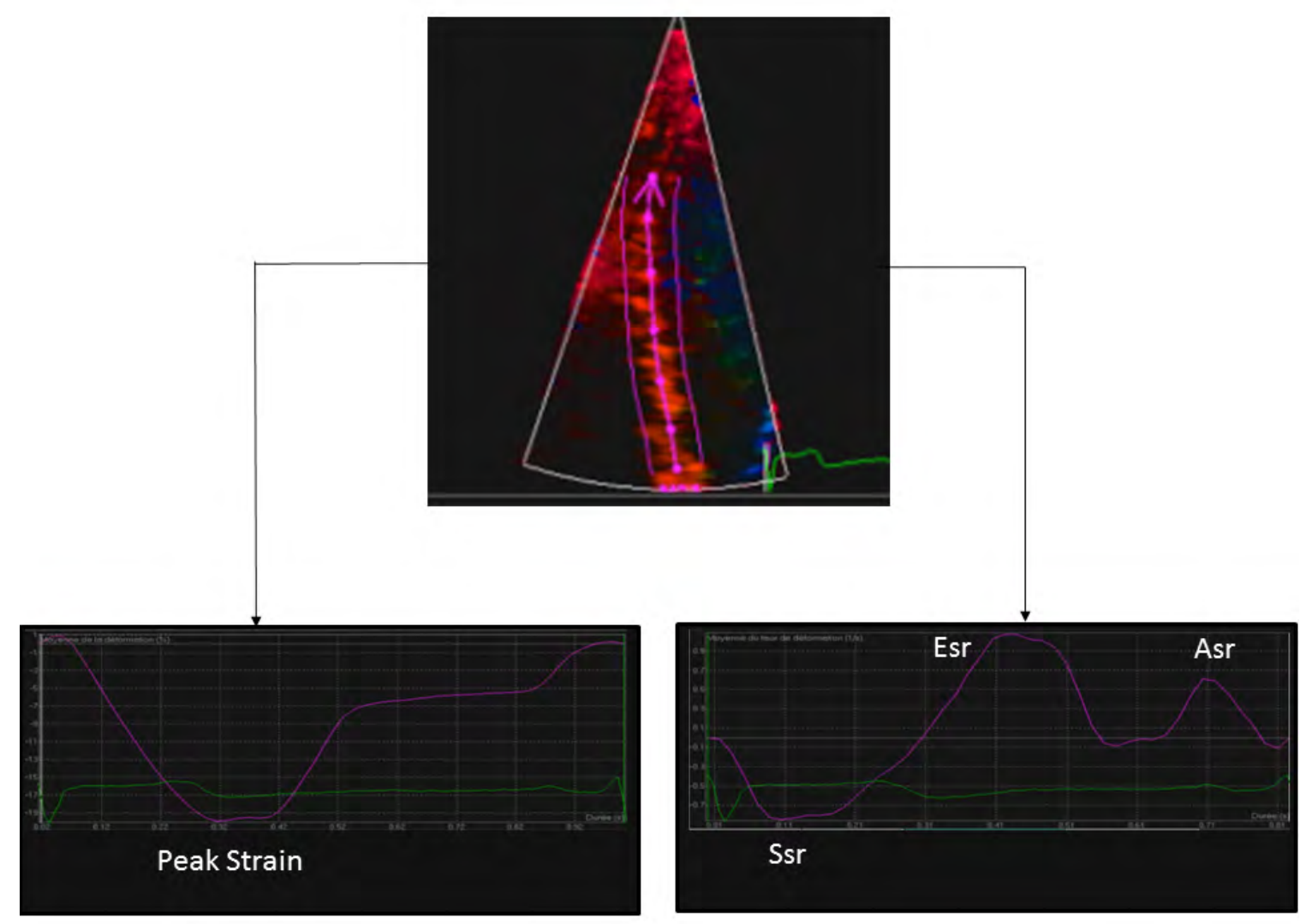

Figure 1: Measurements of peak strain, peak systolic strain rate (Ssr), early peak diastolic strain rate (Esr) and late peak diastolic strain rate (Asr) for the septal wall, by two-dimensional color-coded tissue imaging 
Hodzic et al.: Decreased regional left ventricular myocardial strain in type 1 diabetic children

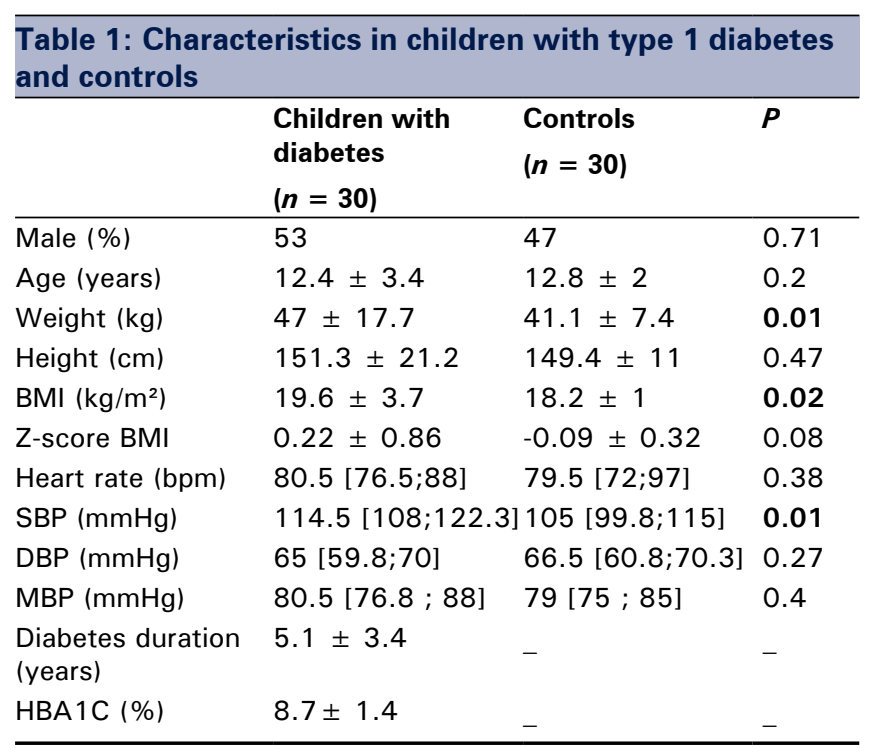

Results are expressed as mean $\pm \mathrm{SD}$, percentage or range. $\mathrm{BMI}$ : body mass index; bpm: beats per minute; SBP/DBP/MBP: systolic/diastolic/ mean blood pressure.

\section{Standard echocardiographic measurements}

The results are shown in Table 2 . In the diabetic group, $Z$ scores for LV-EDD and LV-ESD were significantly lower, while IVS thickness and LVM were significantly higher. The E wave was lower in the diabetic group without reaching statistical significance $(P=0.053)$. There were no significant differences between the two study groups in LVEF and other diastolic function parameters (A wave, E/A and E/ $\mathrm{Ea}$ ) and TDI parameters. St was similar in two study groups.

\section{Strain and strain rate analysis}

Longitudinal strain (Figure 2, Panel A) as well as Ssr (Figure 2 , Panel B) for the septal wall were significantly decreased in patients with diabetes compared with controls. Strain rate diastolic measurements did not differ between the two groups (Figure 1, Panel B). The results are shown in Table 2.

\section{Relation between strain and strain rate parameters, glycemic control and diabetes duration}

Among the longitudinal septal strain parameters, Esr was inversely correlated with $\mathrm{HBA}_{1 \mathrm{C}}(r=-0.41, P=0.028)$. No correlation was found between $\mathrm{HBA}_{1 \mathrm{C}}$ and peak systolic strain $(r=0.26, P=0.16)$ and $\operatorname{Ssr}(r=0.07, P=0.7)$. We did not find any correlation between diabetes duration and strain or strain rate measurements.

\section{DISCUSSION}

Using longitudinal septal strain measurements, we report subclinical left ventricular functional changes in children with type 1 diabetes associated with LV remodeling. Our results suggest that type 1 diabetes is not only a cardiovascular risk factor in the long term but may
Table 2: Echocardiographic characteristics in children with type 1 diabetes and control children

Diabetes children Controls

$(n=30)$

$(n=30)$

\begin{tabular}{|c|c|c|c|}
\hline \multicolumn{4}{|c|}{ 2D standard parameters } \\
\hline LV-EDD (mm) & $42.8 \pm 5.5$ & $43.4 \pm 4.2$ & 0.6 \\
\hline Z-score LV-EDD & $-0.29[-0.89 ; 0.15]$ & $0.17[-0.63 ; 0.8]$ & 0.008 \\
\hline LV-ESD (mm) & $27.3 \pm 3.8$ & $28.5 \pm 3.4$ & 0.16 \\
\hline Z-score LV-ESD & $-0.15 \pm 0.87$ & $0.49 \pm 0.86$ & 0.012 \\
\hline IVS-EDD (mm) & $7.5[6 ; 8.3]$ & $6[5.8 ; 7]$ & 0.002 \\
\hline Z-score IVS-EDD & $0.36[-0.01 ; 0.84]$ & $-0.06[-0.83 ; 0.5]$ & 0.002 \\
\hline PW-EDD (mm) & $7[6 ; 8]$ & $7[6 ; 7]$ & 0.24 \\
\hline Z-score PW-EDD & $0.53[-0.3 ; 1]$ & $0.38[-0.16 ; 0.91]$ & 0.94 \\
\hline LVM $(g)$ & $89[70 ; 129.8]$ & $81[60.8 ; 129]$ & 0.005 \\
\hline $\begin{array}{l}\text { LVM/Height2.7 } \\
(\mathrm{g} / \mathrm{m} 2.7)\end{array}$ & $32.4 \pm 6.5$ & $26.7 \pm 6$ & 0.002 \\
\hline LVEF (\%) & $64[57.8 ; 68.3]$ & $63[59 ; 67.3]$ & 0.97 \\
\hline$E(\mathrm{~cm} / \mathrm{s})$ & $98.8 \pm 13.5$ & $107.9 \pm 20.6$ & 0.053 \\
\hline$A(\mathrm{~cm} / \mathrm{s})$ & $52.5[45.5 ; 61.3]$ & $60[47.8 ; 70.5]$ & 0.1 \\
\hline$E / A$ & $1.9[1.5 ; 2.1]$ & $1.8[1.6 ; 2.1]$ & 0.9 \\
\hline $\mathrm{Ea}(\mathrm{cm} / \mathrm{s})$ & $17.9[15.9 ; 20.1]$ & $18[17 ; 19.3]$ & 0.99 \\
\hline $\mathrm{E} / \mathrm{Ea}$ & $5.5 \pm 1.3$ & $6 \pm 1.6$ & 0.12 \\
\hline St $(\mathrm{cm} / \mathrm{s})$ & $13.2[11.7 ; 15.1]$ & $13[12 ; 14.3]$ & 0.84 \\
\hline \multicolumn{4}{|c|}{ Color TDI analysis } \\
\hline Strain (\%) & $-18.2 \pm 2.4$ & $-20.7 \pm 2.2$ & $<0.001$ \\
\hline $\operatorname{Ssr}(1 / s)$ & $-0.97 \pm 0.22$ & $-1.11 \pm 0.29$ & 0.036 \\
\hline $\operatorname{Esr}(1 / \mathrm{s})$ & $1.52 \pm 0.31$ & $1.55 \pm 0.4$ & 0.84 \\
\hline Asr $(1 / s)$ & $0.72 \pm 0.24$ & $0.81 \pm 0.18$ & 0.1 \\
\hline
\end{tabular}

Values are presented as means \pm SD and range. LV-EDD: left ventricular end-diastolic dimension; IVS-EDD: interventricular septal end-diastolic dimension; LV-PWEDD: left ventricular posterior wall end-diastolic dimension; LVM: left ventricular mass;LVEF: left ventricular ejection fraction; E: mitral early. peak velocity; A: mitral late peak velocity; Ea: mitral annulus early peak velocity;; St: tricuspid peak systolic velocity. Strain : peak strain (\%), Ssr : peak systolic strain rate (1/s); Esr : early peak diastolic peak strain rate (1/s);Asr : late diastolic peak strain rate (1/s)

be associated, early in childhood, with abnormal LV longitudinal function. We did not find a relationship between both glycemic control and diabetes duration and the impairment of LV longitudinal function.

\section{Left ventricular morphology and function}

In our study, children with type 1 diabetes presented an increased LVM. Previous magnetic resonance imaging studies have shown that diabetes was associated with LV hypertrophic remodeling regardless of age and gender ${ }^{[16,17]}$. These results were confirmed by echocardiographic studies where LV remodeling was associated with abnormalities of diastolic function in patients with type 1 diabetes ${ }^{[18,19]}$. Previous echocardiographic studies in diabetic children focused on LV diastolic function and suggested a reduction in early diastolic filling based on transmitral flow analysis ${ }^{[20,21]}$. Although our transmitral Doppler results are consistent with these previous findings, diastolic strain rate parameters failed to identify diastolic dysfunction in the diabetic group. Based on LVEF assessment, all previous studies conducted in children with type 1 diabetes 
A

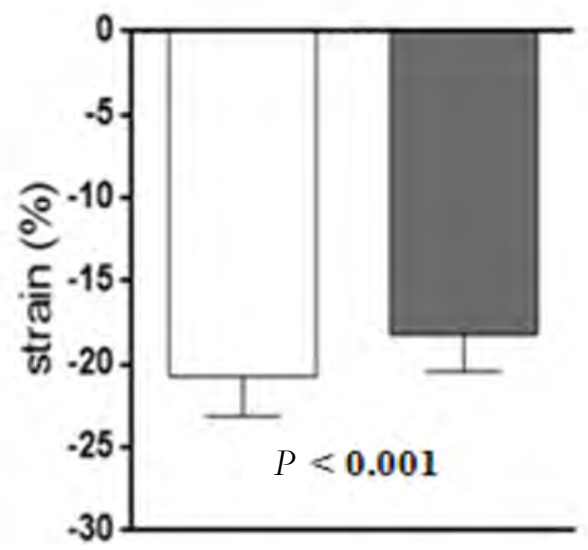

B

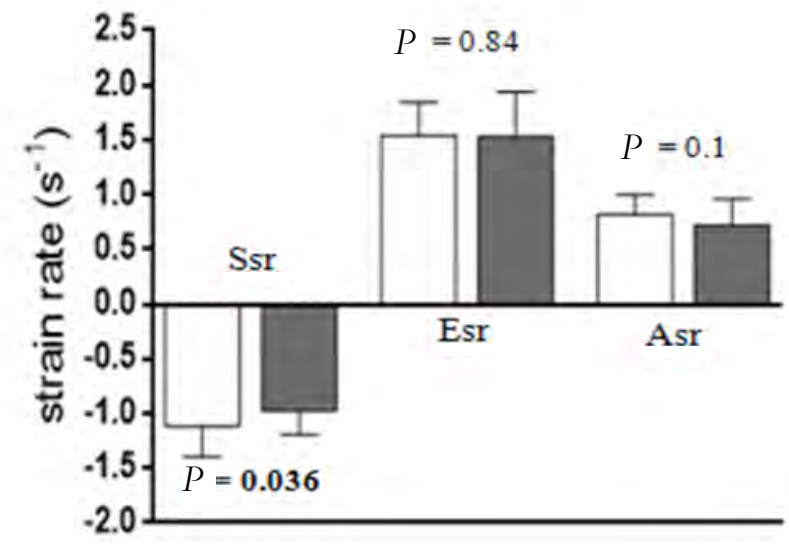

Control

Diabetes
Ssr: systolic peak strain rate; Esr: early diastolic peak stran rate; Asr: late diastolic peak strain rate

Figure 2: Comparison of septal wall strain (Panel A) and strain rate (Panel B) between diabetic patients and controls

concluded the absence of LV systolic dysfunction. Because of cardiac geometry and myofiber arrangements, LV function goes through complex myocardial deformations, or "strains," and cannot be reduced to a variation of volume, as with assessment of LVEF. Longitudinal strain plays an important role in cardiac pump function. It is primarily controlled by subendocardial longitudinal myofibers, which are more susceptible to fibrosis and ischemia $^{[22]}$. This explains why subclinical impairment in longitudinal strain represents the first anomaly observed in the setting of many conditions predisposing to heart failure ${ }^{[23]}$. Our results are consistent with studies in adult diabetic patients, which showed an early impairment of the LV longitudinal strain using $\mathrm{TDI}^{[24]}$ and $2 \mathrm{D}$ speckle strain analysis ${ }^{[25,26]}$, while LVEF was preserved. However, these studies are difficult to interpret because they mixed patients with type 1 and 2 adult diabetic patients, in whom the influence of comorbidities such as age, coronary artery disease, or high blood pressure could not be formally excluded. Such comorbidities may be considered nonexistent in our cohort of diabetic children, who represent a unique model to investigate the early effects of metabolic disturbances induced by type 1 diabetes on myocardial function. Our results are not consistent with the previous studies reported by Salem et al. and Khattab where the LV longitudinal function analyzed by TDI was preserved in children and adolescents with type 1 diabetes ${ }^{[10,27]}$. However, these authors analyzed the velocities of displacement of myocardial walls using pulsed TDI modality, which are more influenced by translational cardiac movements and displacement of adjacent myocardial segments ${ }^{[28]}$. Our results are consistent with those of Abdel-Salam et al. who demonstrated early impairment of LV longitudinal systolic deformation analyzed by $2 \mathrm{D}$ speckle strain in young asymptomatic type 1 diabetic adults without associated comorbidities ${ }^{[2]}$. The consistency of these results suggests that type 1 diabetes may be associated with early longitudinal strain impairment, which may be the first marker of preclinical diabetic cardiomyopathy, as previously suggested in type 2 diabetes ${ }^{[30,31]}$. The strain measurements reflect myocardial deformation, but they are also dependent on load conditions, ventricular geometry, and elastic properties of myocardial tissue ${ }^{[24]}$. In our study, we cannot exclude that the SBP, significantly higher in patients with diabetes, was able to reduce the overall septal strain. However, children with diabetes had a decreased peak systolic strain rate, described as a less dependent parameter of load conditions ${ }^{[32]}$.

\section{Potential mechanisms}

Several pathophysiological mechanisms have been proposed to explain the occurrence of structural and functional myocardial changes observed in diabetic cardiomyopathy ${ }^{[7]}$. These mechanisms include the metabolic abnormalities of cardiomyocytes associated with excessive use of very long chain fatty acids, chronic hyperglycemia, impaired calcium 
homeostasis, fibrosis, myocyte apoptosis, microcirculation abnormalities, and cardiac autonomic dysfunction ${ }^{[5]}$. The decrease in longitudinal deformation may reflect structural and functional changes of myocardial fibers due to diabetes through one of these mechanisms, and may constitute the first sign of diabetic cardiomyopathy ${ }^{[31]}$. A relationship between glycemic control and abnormalities in heart function have been reported in children and adults ${ }^{[33,34]}$. The small number of diabetic children and the relatively short diabetes duration may explain why we did not observe a correlation between glycemic control, diabetes duration, and systolic longitudinal strain in our study.

\section{Clinical perspectives}

Diabetes generates myocardial changes that remain silent during childhood but are expressed in adulthood by excess cardiovascular mortality, mainly attributed to coronary artery disease and heart failure. Endothelial dysfunction and increased arterial stiffness, both established markers of coronary artery atherosclerosis, are already present in children with type 1 diabetes ${ }^{[35]}$. Similarly, our results indicate that type 1 diabetes is associated with early abnormalities of the longitudinal myocardial function. These anomalies could be a first step toward the development of heart failure in adulthood. The presence of early myocardial dysfunction enhances the need for active management of diabetes in children with glycemic control as strict as possible. In addition, LV myocardial strain analysis could help assess the beneficial effect of treatment by objectivizing improved myocardial dysfunction as suggested in adults ${ }^{[33]}$. Further studies are needed to determine the prognostic value of these subclinical abnormalities in heart function and clarify the role of strain in the evaluation and monitoring of diabetic children.

\section{Limitations}

Our work had some limitations. We are aware that the small study population represents a limitation to drawing a formal conclusion. Since we included exclusively Caucasian patients, we cannot extend our results to the whole population of diabetic children. We performed a partial longitudinal strain and strain rate analysis with TDI. We thought this modality may be more appropriate for the children overcoming the difficulties of making an extended ultrasound examination. Furthermore, in children a high-quality apical four-chamber view, necessary for LV strain analysis, may be difficult to obtain, especially for the lateral wall analysis. Finally, being a cross-sectional study, the natural history of the development and progression of altered longitudinal deformation was unknown. Longitudinal clinical follow-up will be required in future studies to determine the prognostic value of longitudinal strain abnormalities.

\section{CONCLUSION}

Our study has suggested that LV longitudinal function is impaired in young patients with T1D. Further follow-up is necessary to elucidate the clinical significance of the myocardial changes detected by $2 \mathrm{D}$ strain imaging in type 1 diabetes children.

\section{Conflict of interest}

None

\section{REFERENCES}

1. Danaei G, Finucane MM, LU Y, Singh GM, Cowan MJ, Paciorek CJ, et al.National, regional andglobal trends in fasting plasma glucose and diabetes prevalence since 1980: systematic analysis of health examination surveys and epidemiological studies with 370 country-years and 2.7 million participants. Lancet 2011;378:31-40.

2. Laing SP, Swerdlow AJ, Slater SD, Botha JL, Burden AC, Waugh NR, et al. The British Diabetic Association Cohort Study, II: cause-specific mortality in patients with insulin-treated diabetes mellitus. Diabet Med 1999;16:466-71.

3. Lind M, Bounias I, Olsson M, Gudbjörnsdottir S, Svensson AM, Rosengren A. Control and incidence of heart failure in 20,985 patients with type 1 diabetes: an observational study. Lancet 2011;378:140-6.

4. Kannel WB, Mc Gee DL. Diabetes and glucose tolerance as risk factors for cardiovascular disease: the Framingham study. Diabetes Care 1979;2:120-6.

5. Fang ZY, Prins JB, Marwick T. Diabetic cardiomyopathy: evidence, mechanisms and therapeutic implications. Endocr Rev 2004;25:543-67.

6. Miki T, Yuda S, Kouzu H. Diabetic cardiomyopathy: pathophysiology and clinical features. Heart Fail Rev 2013;18:149-66.

7. Fang ZY, Schull-Meader R, Leano R. Screening for heart disease in diabetic subjects. Am Heart J. 2005;149:349-54.

8. Boyer JK, Thanigaraj S, Schechtamn KB. Prevalence of ventricular diastolic dysfunction in asymptomatic normotensive patients with diabetes mellitus. Am J Cardiol 2004;93:870-5.

9. Suys B, Katier B, Rooman R, Matthys D, Op De Beeck L, Du Caju MV, et al. Female children and adolescents with type 1 diabetes have morepronounced early echocardiographic signs of diabetic cardiomyopathy. Diabetes Care 2004;27:1947-53.

10. Salem M, El Behery S, Adly A, Khalil D, El Hadidi E. Early predictors of myocardial disease in children and adolescents with type 1 diabetes mellitus. Pediatric Diabetes 2009;10:513-21.

11. Marwick T. Measurement of strain and strain rate by echocardiography: ready for prime time? J Am Coll Cardiol 2006;47:1313-27.

12. Mogelvang R, Sogaard P, Pedersen SA, Olsen NT, Marott JL, Schnohr P et al.Cardiac dysfunction assessed by echocardiographic tissue Doppler imaging is an independent predictor of mortality in the general population. Circulation 2009;119:2679-85.

13. World Health Organization Expert Committee on Diabetes Mellitus. Second report, technical report series. Geneva: WHO; 1980.

14. Rolland-Cachera MF, Cole TJ, Sempé M, Tichet J, Rossignol C, Charraud A. Body mass index variations: centiles from birth to 87 years. Am J Clin Nutr 1991;45:13-21.

15. Labombarda F, Pellissier A, Ellafi M, Creveuil C, Ribault V, Laurans M, et al. Myocardial strain assessment in cystic fibrosis. J Am Soc Echocardiogr 2011;24:1037-45.

16. Velagaleti RS, Gona P, Chuang ML, Salton CJ, Fox CS, Blease SJ, et al. Relations of insulin resistance and glycemic abnormalities to cardiovascular magnetic resonance measures of cardiac structure and function: 
the Framingham Heart Study. Circ Cardiovasc Imaging 2010;3:257-63.

17. Heckbert SR, Post W, Pearson GD, Arnett DK, Gomes AS, JeroschHerold M, et al. Traditional cardiovascular risk factors in relation to left ventricular mass, volume, and systolic function by cardiac magnetic resonance imaging: the Multiethnic Study of Atherosclerosis. J Am Coll Cardiol 2006;48:2285-92.

18. Gul K, Celebi AS, Kacmaz F, Ozcan OC, Ustun I, Berker D, et al. Tissue Doppler imaging must be performed to detect early left ventricular dysfunction in patients with type 1 diabetes mellitus. Eur J Echocardiogr 2009;10:841-6.

19. Nadeau KJ, Regensteiner JG, Bauer TA, Brown MS, Dorosz JL, Hull A et al. Insulin resistance in adolescents with type 1 diabetes and its relationship to cardiovascular function. J Clin Endocrinol Metab 2010;95:513-21.

20. Riggs TW, Transue D. Doppler echocardiographic evaluation of left ventricular diastolic function in adolescents with diabetes mellitus. Am J Cardiol 1990;65:899-902.

21. Gunczler P, Lanes R, Lopez E, Esaa S,Villarroel O, Revel-Chion R. Cardiac mass and function, carotid artery intima-media thickness and lipoprotein (a) levels in children and adolescents with type 1 diabetes mellitus of short duration. J Pediatr Endocrinol Metab 2002;15:181-6.

22. Lumens J, Delhaas T, Arts T, Cowan BR, Young AA. Impaired subendocardial contractile myofiber function in asymptomatic aged humans, as detected using MRI. Am J Physiol Heart Circ Physiol 2006;291:1573-9.

23. Shah AM, Solomon SD. Myocardial deformation imaging: current status and future directions. Circulation 2012;125:e244-8.

24. Fang ZY, Leano R, Marwick T. Relationship between longitudinal and radial contractility in subclinical diabetic heart disease. Clin Sci (Lond); 2004;106:53-60.

25. Ng AC, Delgado V, Bertini M, van der Meer RW, Rijzewijk LJ, Shanks $\mathrm{M}$, et al. Findings from Left Ventricular Strain and Strain Rate Imaging in Asymptomatic Patients With Type 2 Diabetes Mellitus Am J Cardiol 2009;104:1398-401.

26. Zoroufian A, Razmi T, Taghavi-Shavazi M, Lotfi-Tokaldany M, Jalali A. Evaluation of Subclinical Left Ventricular Dysfunction in Diabetic Patients: Longitudinal Strain Velocities and Left Ventricular Dyssynchrony by Two-Dimensional Speckle Tracking Echocardiography Study.
Echocardiography. 2014; 31:456-63.

27. Khattab AA, Soliman MA. Biventricular Function and Glycemic Load in Type 1 Diabetic Children: Doppler Tissue-Imaging Study. Pediatr Cardiol 2015;36:423-31.

28. Tsutsui H, Uematsu M, Shimizu H, Yamagishi M, Tanaka N, Matsuda $\mathrm{H}$ et al. Comparative Usefulness of Myocardial Velocity Gradient in Detecting Ischemic Myocardium by a Dobutamine Challenge. J Am Coll Cardiol. 1998; 31:89-93

29. Abdel-Salam Z, Khalifa M, Ayoub A, Hamdy A, Nammas W. Early changes in longitudinal deformation indices in young asymptomatic patients with type 1 diabetes mellitus: assessment by speckle-tracking echocardiography. Minerva Cardioangiol 2016;64:138-44.

30. Andersson C, Gislason GH, Weeke P, Hoffmann S, Hansen PR, TorpPedersen C, et al. Diabetes is associated with impaired myocardial performance in patients without significant coronary artery disease. Cardiovasc Diabetol 2010;9:3.

31. Ernande L, Bergerot C, Rietzschel ER, De Buyzere ML, Thibault H, Pignonblanc PG, et al. Diastolic dysfunction in patients with type 2 diabetes mellitus: is it really the first marker of diabetic cardiomyopathy? J Am Soc Echocardiogr 2011;24:1268-75.

32. Andersen NH, Terkelsen CJ, Sloth E, Poulsen SH. Influence of preload alterations on parameters of systolic left ventricular long-axis function: A doppler tissue study. J Am Soc Echocardiogr 2004;17: 941-7.

33. Andersen NH, Hansen TK, Christiansen JS. Changes in glycaemic control are related to the systolic function in type 1 diabetes mellitus Scand Cardiovasc J. 2007;41:85-8.

34. Kim EH, Kim YH. Left Ventricular Function in Children and Adolescents With Type 1 Diabetes Mellitus. Korean Circ J 2010;40:125-30.

35. Haller MJ, Stein J, Shuster J, Theriaque D, Silverstein J, Schatz DA. Peripheral artery tonometry demonstrates altered endothelial function in children with type 1 diabetes. Pediatr Diabetes 2007;8:193-8.

How to cite this article: Hodzic A, Ribault V, Maragnes $P$, Milliez $P$, Saloux E, Labombarda F. Decreased regional left ventricular myocardial strain in type 1 diabetic children: A first sign of diabetic cardiomyopathy? J Transl Intern Med 2016; 4: 81-7. 